Article

\title{
Core-Shell-Structured Copolyaniline-Coated Polymeric Nanoparticle Suspension and Its Viscoelastic Response under Various Electric Fields
}

\author{
Il-Jae Moon and Hyoung Jin Choi * \\ Department of Polymer Science and Engineering, Inha University, Incheon 402-751, Korea; \\ E-Mail: 22141656@inha.edu \\ * Author to whom correspondence should be addressed; E-Mail: hjchoi@inha.ac.kr; \\ Tel.: +82-2-860-7486; Fax: +82-2-865-5178.
}

Academic Editor: Luciano Feo

Received: 1 June 2015 / Accepted: 24 July 2015 / Published: 3 August 2015

\begin{abstract}
Semi-conducting poly(n-methylaniline) (PNMA)-coated poly(methyl methacrylate) (PMMA) composite nanoparticles were synthesized using cross-linked and grafted PMMA particles as a core, and then, the PNMA shell was coated via chemical oxidative polymerization on the surface of modified PMMA nanoparticles. Their electroresponsive electrorheological characteristics when dispersed in silicone were confirmed under applied electric fields using a rotational rheometer, focusing on their viscoelastic response. Using a frequency sweep test, the frequency dependence of both the storage and loss moduli was confirmed to increase upon increasing the electric field, with a stable plateau regime over the entire angular frequency range.
\end{abstract}

Keywords: electrorheological; core-shell; viscoelasticity; poly(methyl methacrylate); copolyaniline

\section{Introduction}

Electrorheological (ER) fluids are typically prepared by dispersing polarizable or semiconducting particles in an insulating liquid media. When various electric fields are applied, the state of the ER fluid is changed reversibly from liquid-like to solid-like because of the formation of fibril-chain structures. This change occurs because the chains oriented along the electric field direction form under an applied 
electric field [1-3]. The transition of structures changes its rheological properties, including the shear stress, shear viscosity, yield stress $\left(\tau_{y}\right)$, storage modulus $\left(G^{\prime}\right)$, and loss modulus $\left(G^{\prime \prime}\right)$.

The viscoelastic behavior of ER fluids under various electric field strengths is associated with the induced particle structures, which can be observed in an oscillatory dynamic experiment. In this case, the viscoelasticity is the material characteristic representing both the elastic and viscous properties. In other words, viscoelastic materials are capable of balancing the strong part of the input energy and dissipating the remainder of the energy under constant deformation [4]. However, the rheological properties of an ER fluid are dependent on the measuring range, such as the pre-yield and yield region. Because the ER suspensions dominantly represent elasticity in the pre-yield region, the linear viscoelasticity can be adopted. In contrast, non-linear viscoelasticity is observed in the yield region [5].

The distinctive advantages of ER fluids include their short reaction time, simple mechanics, and low energy consumption [6,7], and under an external electric field, these characteristics are similar to those of magnetorheological (MR) fluids under an applied external magnetic field [8]. Because of their controllable and reversible transition, ER fluids have been extensively applied for a wide range of electromechanical engineering devices such as isolation dampers, shock absorbers, and clutches as well as for engine efficiency [9-11]. Therefore, studies on electroresponsive materials have attracted significant attention in the search for suitable candidates for ER suspensions, such as inorganics [12-14] with high dielectric properties, inorganic/organic composites [15,16], and organic or polymeric semiconducting materials [17-21]. Importantly, the electrical conductivity of ER fluids must be in the semiconducting region to avoid the short circuit of the rheometer during ER experiments under a high applied voltage [22].

In contrast, in a core/shell-structured system, not only can the particle size and density be controlled, it is also possible to produce desired materials that are functionalized via a single or multiple coating process. This core/shell-structured system has been reported to exhibit an enhanced ER effect [23,24]. Poly(methyl methacrylate) (PMMA) has been used as a core material extensively because of its excellent characteristics such as monodispersity and spherical microspheres.

Among the range of ER materials, polyaniline (PANI) possesses excellent advantageous characteristics such as its facile synthesis, tunable conductivity with a dedoping and doping process, and good environmental stability [25-27]. Nevertheless, PANI exhibits poor processability because of its brittleness and poor compatibility due to its insolubility in most organic solvents. To overcome these problems, as a derivative of PANI, poly(N-methylaniline) (PNMA) has received much attention owing to its higher oxidation stability and improved solubility in organic solvents. In addition, its relatively low electrical conductivity compared with PANI can be readily controlled using a dedoping process [28,29].

Both PNMA and PANI can be synthesized via a chemical oxidative polymerization method, which is known to be the most widely adopted synthetic route for conducting PANI. The aniline constitutional units are connected head to tail in the para positions, with half of the nitrogen being the secondary amine type, and the other half being the imine type. The PANI is generally produced as a protonated green and conducting emeraldine salt. However, depending on the oxidation state, the fully oxidized form of emeraldine, pernigraniline, could be an important polymerization intermediate; the reduced form of emeraldine, leucoemeraldine, is the other non-conducting form of PANI [30,31]. Note that the oxidative polymerization of PANI also depends on the reaction conditions, including the chemical nature of the 
oxidants, concentrations of the reactants such as oxidant and aniline, solvent components, and the presence of additives including stabilizers and surfactants.

In this study, core/shell-structured PMMA-PNMA particles were synthesized using a novel grafting polymerization process. The PMMA seeds were swollen using glycidyl methacrylate (GMA), and ethylene glycol dimethacrylate (EGDMA) was added as a cross-linking agent. Then, the core/shell interaction was enhanced by an epoxy-amine reaction between GMA and oxydianiline (ODA). In our previous experiments, the typical ER performance of a PMMA-PNMA particle-based ER fluid was analyzed using a rotational rheometer in rotation mode [32]. However, in this experiment, the ER properties of the obtained PMMA-PNMA particles were confirmed using a rotational rheometer in oscillatory mode under various applied electric field strengths.

\section{Experiments}

\subsection{Materials and Nanoparticle Fabrication}

Both the $\alpha, \alpha^{\prime}$-azobisisobutyronitrile (AIBN) (98\% purity, Junsei Chemical, Tokyo, Japan) and methyl methacrylate (Sigma-Aldrich, St. Louis, MO, USA) were purified before use. The other chemical reagents, including benzoyl peroxide (BPO), glycidyl methacrylate (GMA) (97\% purity, Sigma-Aldrich), poly(vinylpyridine) (PVP) ( $\mathrm{w} \mathrm{w}=360,000 \mathrm{~g} / \mathrm{mol}$, Sigma-Aldrich), EGDMA (98\% purity, Sigma-Aldrich), ammonium persulfate (APS) (Daejung chemical, Siheung, Korea), ODA (97\% purity, Sigma-Aldrich), sodium dodecylsulfate (SDS) (Acros Organics, Seoul, Korea), poly(vinyl alcohol) (PVA 1700) (DC Chemical, Seoul, Korea), and NMA (Tokyo Chemical Industry, Tokyo, Japan) were used as received.

Initially, the PMMA nanoparticles were synthesized using dispersion polymerization. A MMA monomer and PVP as a stabilizer were dispersed in methanol with a radical initiator (AIBN) at room temperature. The polymerization was allowed to proceed at $65^{\circ} \mathrm{C}$ for $24 \mathrm{~h}$ under continuous stirring. The obtained product was collected by centrifugation, washed with methanol and distilled water, and then dried using a freeze-drier.

The obtained PMMA particles were then dispersed in deionized water containing SDS and swollen using GMA with a radical initiator for $12 \mathrm{~h}$ at room temperature. In the course of mild stirring, the SDS was adsorbed on the PMMA surface. The mixture of BPO and EGDMA was placed into the reactor. In this case, EGDMA was used as a crosslinking agent. Then, GMA dissolved in the SDS aqueous solution was added to swell the above mixture for $6 \mathrm{~h}$. The obtained swollen and crosslinked particles (PMMGMA) were then dispersed in acetone containing ODA. The epoxy-amine reaction between glycidyl groups in the GMA and amine groups in the ODA was carried out at $55{ }^{\circ} \mathrm{C}$ for $12 \mathrm{~h}$, followed by centrifugation and drying of the ODA-PMMGMA particles.

The synthesized ODA-PMMGMA particles were dissolved in an acidic medium containing PVA as the stabilizer and APS as the initiator. The chemically oxidative polymerization was progressed by adding NMA as the monomer and $\mathrm{HCl}$ as the oxidant at $0{ }^{\circ} \mathrm{C}$ for $24 \mathrm{~h}$. The final product was collected via centrifuge and then dried for $24 \mathrm{~h}$ at $55^{\circ} \mathrm{C}$. Figure 1 shows the detailed experimental mechanism from PMMA seeds to PMMA/PNMA microspheres, and more details on the synthesis can be found in the previous report [32]. 


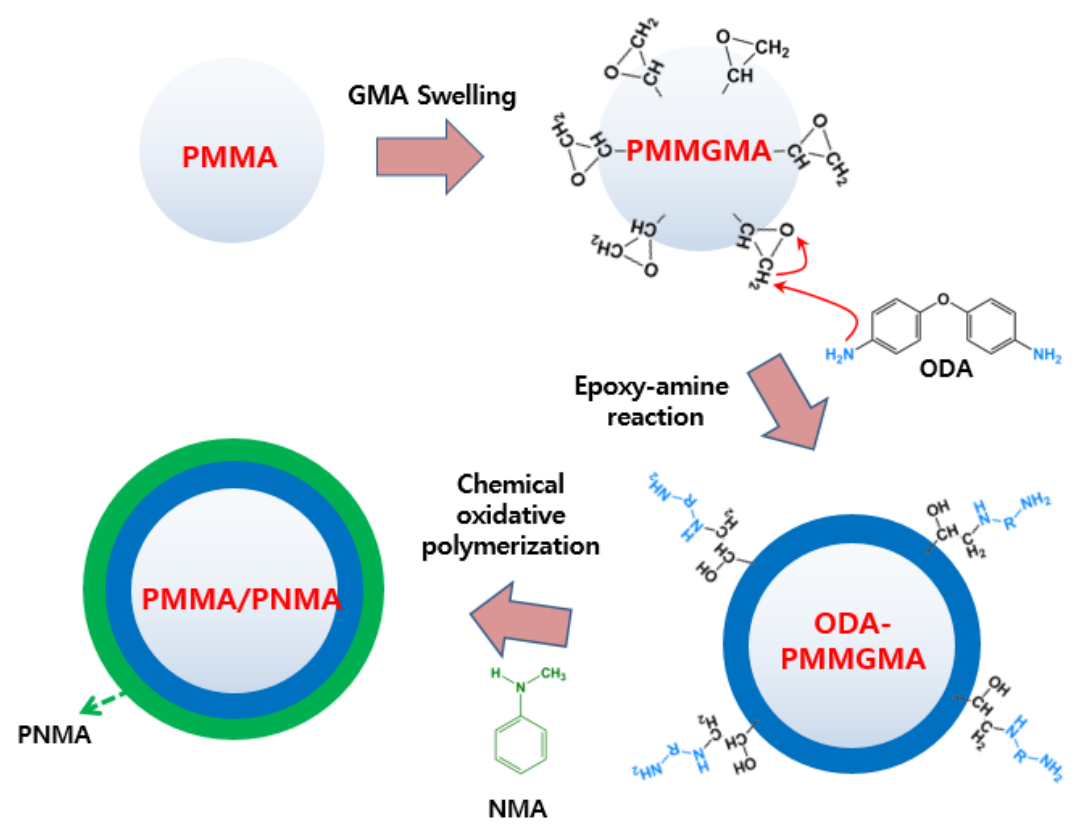

Figure 1. Schematic diagram illustrating the experimental route used to fabricate Semi-conducting PMMA/PNMA particles.

The fabricated PMMA-PNMA particles were then subjected to a dedoping process to reduce their conductivity from $9.319 \times 10^{-4}$ to $5.35 \times 10^{-10} \mathrm{~S} / \mathrm{cm}$, which is within the semiconducting regime. The PMMA-PNMA particles were then dispersed in silicone oil with a volume fraction of $10 \%$ via ultrasonication to achieve good dispersion.

\subsection{Characterization}

The morphology of the synthesized particles was examined using field-emission scanning electron microscopy (FE-SEM) (S-4300, Hitachi, Tokyo, Japan) at a voltage of $15 \mathrm{kV}$ and a working distance of $15 \mathrm{~mm}$. The viscoelastic properties of the PMMA-PNMA particle-based ER fluid were examined using a rotational rheometer (Physica MCR 300, Stuttgart, Germany) equipped with a high-voltage generator (HCP 7E -12500, fug, Schechen, Germany) using a Couette-type sample loading geometry with a bob and cup (CC17, gap size is $0.71 \mathrm{~mm}$ ) under a dynamic oscillation test.

\section{Result and Discussion}

The SEM images were used to determine the surface morphology and particle size. As observed in Figure 2a, the surface of the fabricated PMMA nanoparticles appears smooth with a uniform diameter of $700 \mathrm{~nm}$. However, for the PMMA-PNMA particles in Figure 2b, the particle surface was considerably rough and coated irregularly, with the average particle size being increased to $1.63 \mu \mathrm{m}$. This very distinctive difference in their morphology indicates successful coating of the PNMA.

In our previous experiments, the chemical structure of the fabricated PMMA-PNMA microspheres was examined using FT-IR spectroscopy [32]. All the characteristic peaks not only for PMMA and PMMA-PNMA but also for the intermediate steps of PMMGMA and ODA-PMMGMA were carefully identified. The successful fabrication of the core-shell-structured PMMA-PNMA microspheres was then confirmed. 
Dynamic-oscillatory testing using a rotational rheometer is an important tool for studying the viscoelastic properties of ER fluids. Initially, the amplitude sweep test was performed to verify the limit of the linear viscoelastic range $\left(\gamma_{\mathrm{LVE}}\right)$ with a fixed angular frequency of $6.28 \mathrm{rad} \cdot \mathrm{s}^{-1}$ before the main dynamic oscillation test. Figure 3 shows the change in both $G^{\prime}$, which represents the elastic storage response during the shear process, and $G^{\prime \prime}$, which represents the energy dissipation response during the shear process (viscous property) as a function of the strain from $1 \times 10^{-5}$ to 1 . $G^{\prime}$ is much larger than $G^{\prime \prime}$ at the relevant electric field strength. In addition, the $G^{\prime}$ and $G^{\prime \prime}$ curves exhibited a constant plateau value in the low-amplitude region, which is the so-called $\gamma_{\mathrm{LVE}}$. In the $\gamma_{\mathrm{LVE}}$ region, deformation of the structure is considered reversible, and the elasticity term is dominant compared to the viscosity term. The mean

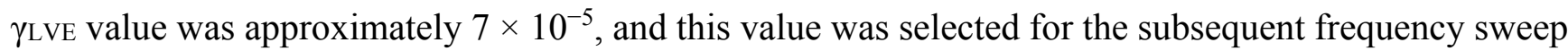
test. When the applied strain amplitude exceeds the value of $\gamma_{\mathrm{LVE}}$, both $G^{\prime}$ and $G^{\prime \prime}$ decrease sharply because of the irreversible change in the structure, and the value of $G^{\prime \prime}$ even exceeds that of $G^{\prime}$.

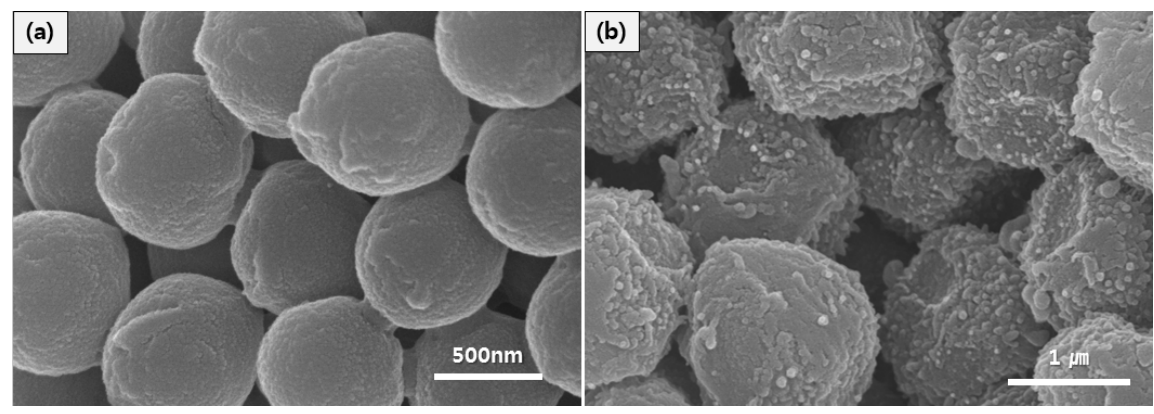

Figure 2. Scanning electron microscopy (SEM) images of (a) pure PMMA seeds and (b) PMMA-PNMA particles.

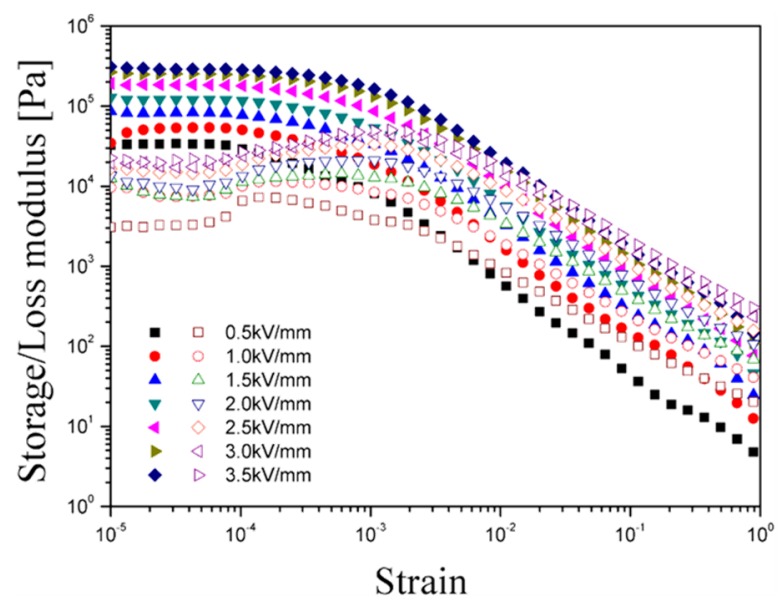

Figure 3. Amplitude sweep of the PMMA-PNMA particle-based electrorheological (ER) fluid (closed symbols for $G^{\prime}$, open symbols for $G^{\prime \prime}$ ).

Examining the magnitude of the in-phase (elastic) component of stress $\left(\tau^{\prime}=G^{\prime} \gamma\right)$ as a function of strain $\gamma$ is a useful way to illustrate the progressive structural breakdown, as illustrated in Figure 4 . The shoulders or maximum values of the elastic stress provide a good estimation of the localization of $\tau_{\mathrm{y}}$. This approach has been used to explain the existence of two yielding points and progressive structural destruction. At small strain amplitudes $(\gamma<0.001)$, the in-phase stress increases linearly with the strain within the linear viscoelastic region. The structure of the PMMA-PNMA particle-based ER fluid began 
to break down at the limiting strain $\left(\gamma_{c}\right) . \gamma_{c}$ represents the breaking of intermolecular bonds in the network of the ER fluid [33-36].

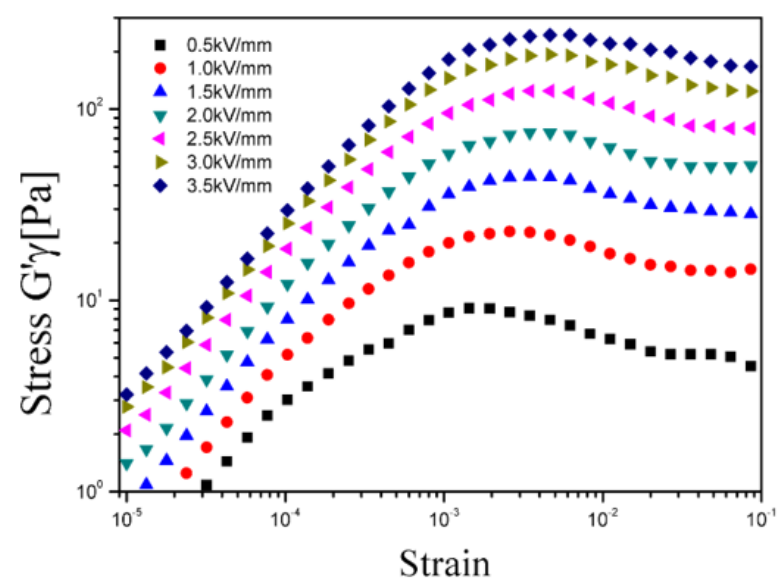

Figure 4. In-phase stress component $\left(G^{\prime} \gamma\right)$ as a function of strain replotted using the data presented in Figure 3.

The dependence of $\tau_{\mathrm{y}}$ on the applied electric field strength is analyzed in Figure 5. Typically, under an applied electric field, the correlation between $\tau_{\mathrm{y}}$ and the electric field strength is investigated using the following power-law equation, similar to either the static yield stress or dynamic yield stress:

$$
\tau_{\mathrm{y}} \propto E^{m}
$$

where $m=2.0$ is suggested by the polarization model and $m=1.5$ is indicated for the conduction model [37]. In Figure 5, all the experimental points for $\tau_{\mathrm{y}}$ fall along a straight line with a slope of 1.5. Thus, $\tau_{\mathrm{y}}$ of this ER suspension is regarded to follow the conduction model.

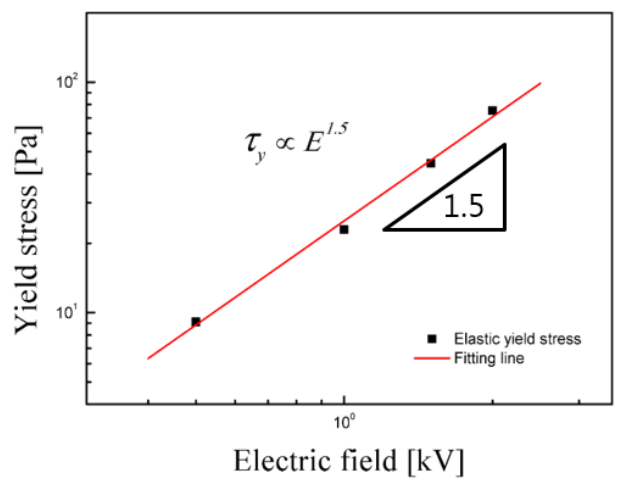

Figure 5. $\tau_{\mathrm{y}}$ versus electric field strength of the ER fluid containing 10 vol \% PMMA-PNMA particles.

The behavior of $G^{\prime}$ and $G^{\prime \prime}$ as a function of the angular frequency were further examined at various electric field strengths based on the selected critical strain $\left(7 \times 10^{-5}\right)$, as shown in Figure 6. By increasing the electric field, both $G^{\prime}$ and $G^{\prime \prime}$ increased with a stable plateau regime for of the entire angular frequency range, indicating the frequency independence. Furthermore, $G^{\prime}$ was always higher than $G^{\prime \prime}$, indicating the dominancy of elastic versus viscous behavior in the structure of the ER fluid. Namely, the ER fluid exhibits good solid-like behavior in an electric field. 


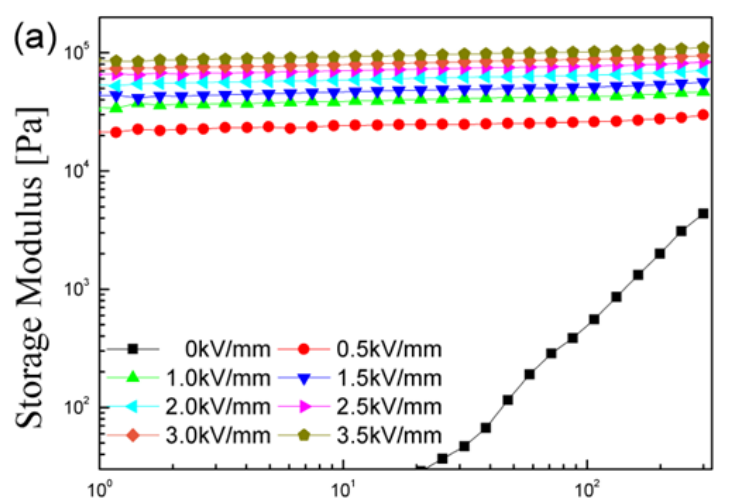

Angular Frequency $[\mathrm{rad} / \mathrm{s}]$

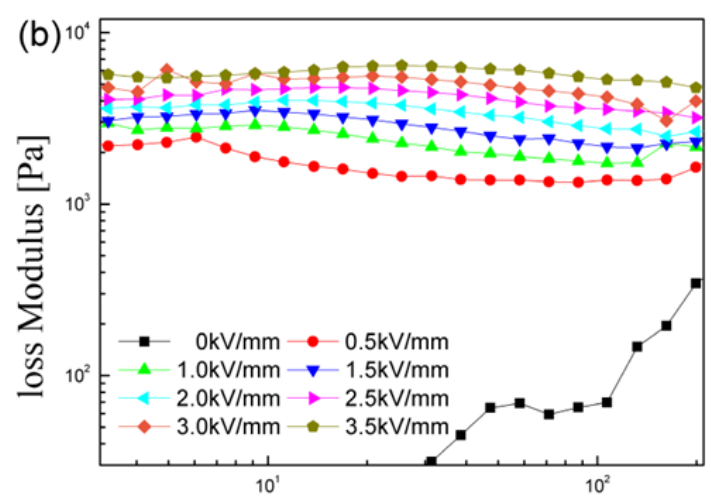

Angular Frequency $[\mathrm{rad} / \mathrm{s}]$

Figure 6. Angular frequency sweep versus $G^{\prime}(\mathbf{a})$ and $G^{\prime \prime}(\mathbf{b})$ with a fixed strain amplitude of $7 \times 10^{-5}$ for the $10 \mathrm{vol} \%$ PMMA-PNMA particle-based ER fluid under various electric field strengths.

In addition, the complex viscosity $\left(\eta^{*}\right)$ of the PMMA-PNMA particle-based ER suspension was further analyzed as a function of frequency at various electric field strengths, as shown in Figure 7. Without an applied electric field, $\eta^{*}$ is hardly dependent on the frequency. However, under an electric field, the complex viscosity decreases as the frequency increases over the entire frequency region. In addition, when the electric field is increased, the increase of particle-particle interaction in the ER fluid leads to a higher complex viscosity over the entire frequency range [38].

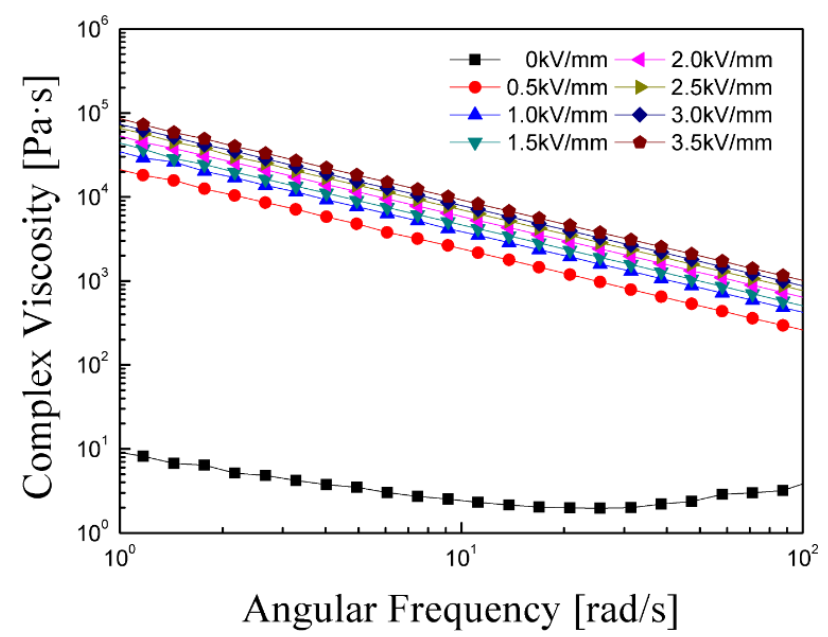

Figure 7. Complex viscosity $\left(\eta^{*}\right)$ of PMMA-PNMA particle-based ER fluid as a function of angular frequency.

The ER efficiency is an important factor to assess the changes in the behavior of the ER system with and without the external electric field and is calculated using the following equation:

$$
e=\frac{\left(\eta^{*}-\eta_{0}\right)}{\eta_{0}}
$$

where $\eta^{*}$ is the complex viscosity in the presence of an electric field and $\eta_{0}$ is the field-off complex viscosity. Figure 8 shows the dependence of the ER efficiency on the angular frequency. The ER efficiency increased with increasing electric field strength [39]. 


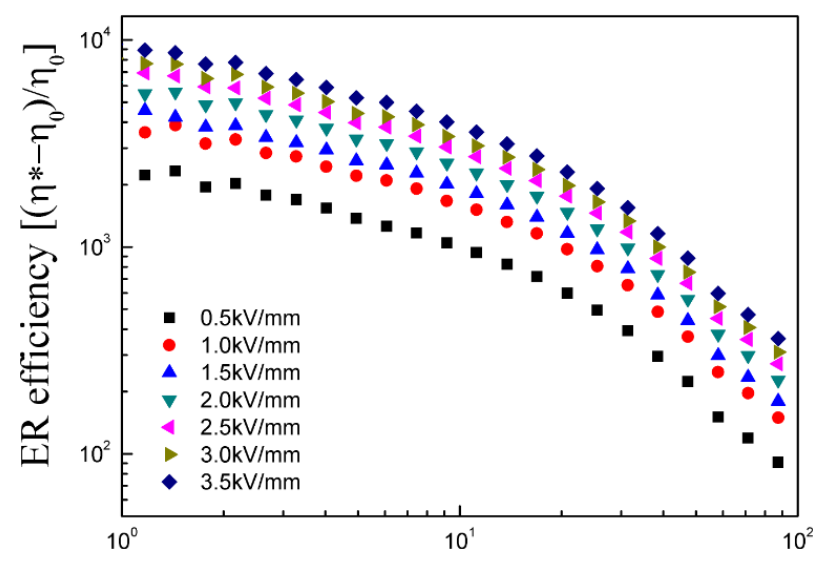

Angular Frequency $[\mathrm{rad} / \mathrm{s}]$

Figure 8. ER efficiency as a function of angular frequency of PMMA-PNMA particle-based ER fluid.

The solid-like characteristic of the ER fluids could also be interpreted by examining the stress relaxation behavior. Figure 9 shows the stress relaxation modulus $G(t)$ results, which were calculated from the values of $\mathrm{G}^{\prime}$ and $\mathrm{G}^{\prime \prime}$ using the frequency data given in Figure 6 and the numerical formula given in Equation (3):

$$
G(t) \approx G^{\prime}(\omega)-0.560 G^{\prime \prime}(\omega / 2)+0.200 G^{\prime \prime}(\omega)
$$

This equation is called the Schwarzl equation [40,41], and $G(t)$ in Figure 9 shows plateau behavior upon increasing the electric field on a logarithmic scale as a function of time. In addition, the Schwarzl equation proves the extremely short-term relaxation behavior of the PMMA-PNMA, which is very difficult to determine experimentally because of the weakness of the mechanical measurement caused by the device itself as well as the intrinsic properties of the ER materials. Figure 9 indicates that under an external electric field, $G(t)$ had a plateau region unlike $G(t)$ without an electric field. Hence, the ER fluid of PMMA-PNMA exhibits a distinctive solid-like behavior under an external electric field because of the strong attractive interactions between the particles.

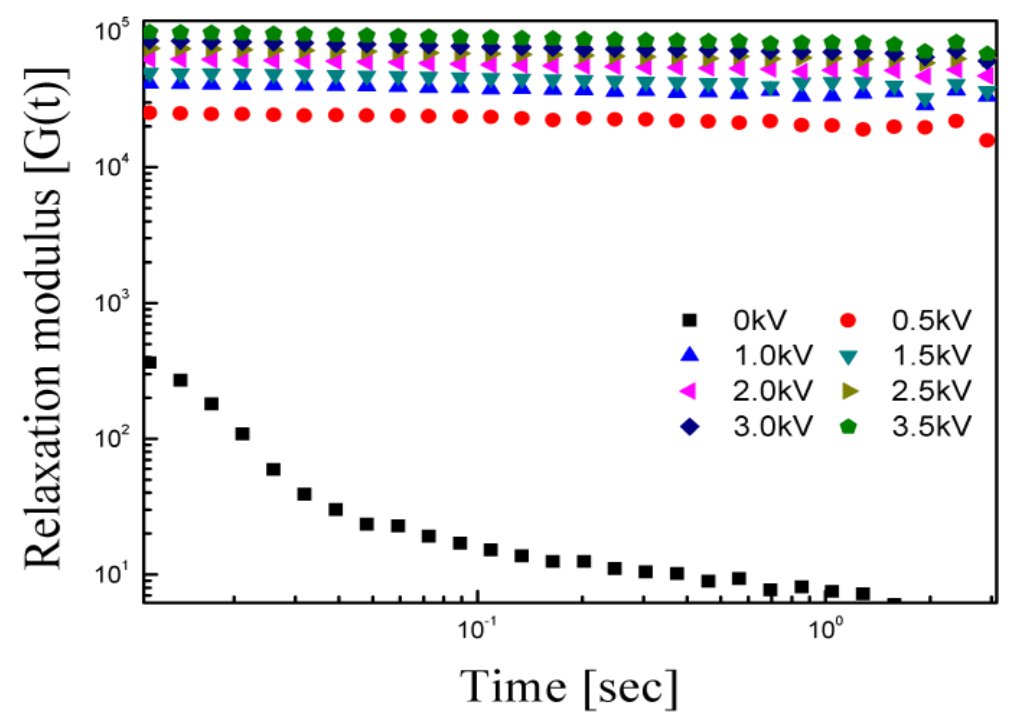

Figure 9. Relaxation modulus of the ER fluids calculated from $G^{\prime}$ and $G^{\prime \prime}$. 


\section{Conclusions}

Core-shell-structured PMMA-PNMA microspheres were successfully prepared by adopting a monodispersed PMMA core and PNMA shell, and the PMMA-PNMA particle-based ER fluid exhibited a viscoelastic ER response in the dynamic oscillation tests under various applied electric field strengths. The slope of 1.5 for the yield stress obtained from the elastic stress indicated that the ER system follows a conduction model. Furthermore, along with drastic increases in the ER efficiency, distinctive changes from a liquid-like to a solid-like phase were observed based on the viscoelastic characteristics of the dynamic moduli and complex viscosity. Using the dynamic moduli data, we also obtained shear relaxation modulus estimated from the Schwarzl equation. Not only the phase change from a liquid-like state to a solid-like state, but also plateau shear modulus increase with increased electric fields was further observed.

\section{Acknowledgments}

This work was supported by a research grant from the National Research Foundation, Korea (NRF-2013R1A1A2057955).

\section{Author Contributions}

Il-Jae Moon performed experiments and analyzed the data. Hyoung Jin Choi supervised the project and the preparation of the manuscript.

\section{Conflicts of Interest}

The authors declare no conflict of interest.

\section{References}

1. Yin, J.B.; Xia, X.A.; Xiang, L.Q.; Zhao, X.P. Coaxial cable-like polyaniline@titania nanofibers: Facile synthesis and low power electrorheological fluid application. J. Mater. Chem. 2010, 20, 7096-7099.

2. Cheng, Q.L.; Pavlinek, V.; He, Y.; Yan, Y.F.; Li, C.Z.; Saha, P. Synthesis and electrorheological characteristics of sea urchin-like TiO2 hollow spheres. Colloid Polym. Sci. 2011, 289, 799-805.

3. Cabuk, T.Z.; Sari, B.; Unal, H.I. Preparation of novel polyindene/polyoxymethylene blends and investigation of their properties. J. Appl. Polym. Sci. 2010, 117, 3659-3664.

4. Cho, M.S.; Lee, J.H.; Choi, H.J.; Ahn, K.H.; Lee, S.J.; Jeon, D. Linear viscoelasticity of semiconducting polyaniline based electrorheological suspensions. J. Mater. Sci. 2004, 39, 1377-1382.

5. Martin, J.E.; Adolf, D.; Halsey, T.C. Electrorheology of a model colloidal fluid. J. Colloid Interf. Sci. 1994, 167, 437-452.

6. Liu, Y.D.; Park, B.J.; Kim, Y.H.; Choi, H.J. Smart monodisperse polystyrene/polyaniline core-shell structured hybrid microspheres fabricated by a controlled releasing technique and their electro-responsive characteristics. J. Mater. Chem. 2011, 21, 17396-17402.

7. Hiamtup, P.; Sirivat, A.; Jamieson, A.M. Strain-hardening in the oscillatory shear deformation of a dedoped polyaniline electrorheological fluid. J. Mater. Sci. 2010, 45, 1972-1976. 
8. de Vicente, J.; Klingenberg, D.J.; Hidalgo-Alvarez, R. Magnetorheological fluids: A review. Soft Matter 2011, 7, 3701-3710.

9. Stanway, R.; Sproston, J.L.; El-Wahed, A.K. Applications of electro-rheological fluids in vibration control: A survey. Smart Mater. Struct. 1996, 5, doi:10.1088/0964-1726/5/4/011.

10. Gumus, O.Y.; Erol, O.; Unal, H.I. Polythiophene/borax conducting composite II: Electrorheology and industrial applications. Polym. Compos. 2011, 32, 756-765.

11. Khanicheh, A.; Mintzopoulos, D.; Weinberg, B.; Tzika, A.A.; Mavroidis, C. Evaluation of electrorheological fluid dampers for applications at 3-T MRI environment. IEEE ASME Mechatron. 2008, 13, 286-294.

12. Plachy, T.; Mrlik, M.; Kozakova, Z.; Suly, P.; Sedlacik, M.; Pavlínek, V.; Kuritka, I. The electrorheological behavior of suspensions based on molten-salt synthesized lithium titanate nanoparticles and their core-shell titanate/urea analogues. ACS Appl. Mater. Interfaces 2015, 7, 3725-3731.

13. Tian, X.L.; Yin, Y.C.; Wang, B.X.; Song, X.F.; Sun, S.S.; Yu, S.S.; Hao, C.C.; Chen, K.Z. Anisotropic $\alpha-\mathrm{Fe}_{2} \mathrm{O}_{3} @ \mathrm{TiO}_{2}$ core-shell nanoparticles and their smart electrorheological response. Eur. J. Inorg. Chem. 2015, 2015, 430-440.

14. Qiao, X.X.; Luo, Y.X.; Sun, A.H.; Wang, C.Y.; Zhang, J.F.; Chu, C.Y.; Guo, J.J.; Xu, G.J. Effect of surface modification of $\mathrm{SiO}_{2} @ \mathrm{TiO}_{2}$ core-shell particles on the structural colour under an electric field. $R S C$ Adv. 2015, 5, 6489-6493.

15. Yin, J.B.; Wang, X.X.; Zhao, X.P. Silicone-grafted carbonaceous nanotubes with enhanced dispersion stability and electrorheological efficiency. Nanotechnology 2015, 26, doi:10.1088/ 0957-4484/26/6/065704.

16. Oh, S.Y.; Kang, T.J. Electrorheological response of inorganic-coated multi-wall carbon nanotubes with core-shell nanostructure. Soft Matter 2014, 10, 3726-3737.

17. Yin, J.B.; Zhao, X.P.; Xiang, L.Q.; Xia, X.; Zhang, Z.S. Enhanced electrorheology of suspensions containing sea-urchin-like hierarchical Cr-doped titania particles. Soft Matter 2009, 5, 4687-4697.

18. Hong, J.Y.; Jang, J. Highly stable, concentrated dispersions of graphene oxide sheets and their electro-responsive characteristics. Soft Matter 2012, 8, 7348-7350.

19. Yoon, D.J.; Kim, Y.D. Electrorheological properties of polypyrrole-SnO 2 -methylcellulose nanocomposite suspensions. J. Mater. Sci. 2007, 42, 5534-5538.

20. Fang, F.F.; Choi, H.J.; Joo, J. Conducting polymer/clay nanocomposites and their applications. J. Nanosci. Nanotechnol. 2008, 8, 1559-1581.

21. Choi, H.J.; Kim, J.W.; To, K.W. Electrorheological characteristics of semiconducting poly(aniline-co-o-ethoxyaniline) suspension. Polymer 1999, 40, 2163-2166.

22. Zhang, W.L.; Choi, H.J. Dynamic response of a graphene oxide-polyaniline composite suspension under an applied electric field. J. Korean Phys. Soc. 2012, 61, 1422-1425.

23. Pavlínek, V.; Sáha, P.; Kitano, T.; Stejskal, J.; Quadrat, O. The effect of polyaniline layer deposited on silica particles on electrorheological and dielectric properties of their silicone-oil suspensions. Phys. A Stat. Mech. Appl. 2005, 353, 21-28.

24. Hwang, J.K.; Shin, K.; Lim, H.S.; Cho, J.C.; Kim, J.W.; Suh, K.D. The effects of particle conductivity on the electrorheological properties of functionalized MCNT-coated doublet-shaped anisotropic microspheres. Macromol. Res. 2012, 20, 391-396. 
25. Quadrat, O.; Stejskal, J. Polyaniline in electrorheology. J. Ind. Eng. Chem. 2006, 12, 352-361.

26. Sedlačík, M.; Mrlík, M.; Pavlínek, V.; Sáha, P.; Quadrat, O. Electrorheological properties of suspensions of hollow globular titanium oxide/polypyrrole particles. Colloid Polym. Sci. 2012, 290, $41-48$.

27. Cheng, Q.L.; Pavlinek, V.; He, Y.; Li, C.Z.; Saha, P. Electrorheological characteristics of polyaniline/titanate composite nanotube suspensions. Colloid Polym. Sci. 2009, 287, 435-441.

28. Planes, G.A.; Rodriguez, J.L.; Miras, M.C.; Pastor, E.; Barbero, C.A. Spectroelectrochemical studies of poly(N-methylaniline) formation, redox behaviour and degradation. A comparison with polyaniline. Electrochim. Acta 2014, 122, 39-49.

29. Harima, Y.; Sanada, K.; Patil, R.; Ooyama, Y.; Mizota, H.; Yano, J. Monodisperse and isolated microspheres of poly(N-methylaniline) prepared by dispersion polymerization. Eur. Polym. J. 2010, $46,1480-1487$.

30. Sapurina, I.; Stejskal, J. The mechanism of the oxidative polymerization of aniline and the formation of supramolecular polyaniline structures. Polym. Int. 2008, 57, 1295-1325.

31. Gospodinova, N.; Terlemezyan, L.; Mokreva, P.; Kossev, K. On the mechanism of oxidative polymerization of aniline. Polymer 1993, 34, 2434-2437.

32. Moon, I.J.; Kim, H.Y.; Choi, H.J. Conducting poly(N-methylaniline)-coated cross-linked poly(methyl methacrylate) nanoparticle suspension and its steady shear response under electric fields. Colloids Surf. A Physicochem. Eng. Asp. 2015, 481, 506-513.

33. Fernández-Toledano, J.C.; Rodriguez-López, J.; Shahrivar, K.; Hidalgo-Álvarez, R.; Elvira, L.; de Espinosa, F.M.; de Vicente, J. Two-step yielding in magnetorheology. J. Rheol. 2014, 58, 1507-1534.

34. Segovia-Gutiérrez, J.P.; Berli, C.L.A.; de Vicente, J. Nonlinear viscoelasticity and two-step yielding in magnetorheology: A colloidal gel approach to understand the effect of particle concentration. J. Rheol. 2012, 56, 1429-1448.

35. Pan, X.D.; McKinley, G.H. Structural limitation to the material strength of electrorheological fluids. Appl. Phys. Lett. 1997, 71, 333-335.

36. Chin, B.D.; Winter, H.H. Field-induced gelation, yield stress, and fragility of an electro-rheological suspension. Rheol. Acta 2002, 41, 265-275.

37. Méheust, Y.; Parmar, K.P.S.; Schjelderupsen, B.; Fossum, J.O. The electrorheology of suspensions of Na-fluorohectorite clay in silicone oil. J. Rheol. 2011, 55, 809-833.

38. Zhang, K.; Park, B.J.; Fang, F.F.; Choi, H.J. Sonochemical preparation of polymer nanocomposites. Molecules 2009, 14, 2095-2110.

39. Trlica, J.; Sáha, P.; Quadrat, O.; Stejskal, J. Electrical and electrorheological behavior of poly(aniline-co-1,4-phenylenediamine) suspensions. Eur. Polym. J. 2000, 36, 2313-2319.

40. Prasad, R.; Pasanovic-Zujo, V.; Gupta, R.K.; Cser, F.; Bhattacharya, S.N. Morphology of EVA based nanocomposites under shear and extensional flow. Polym. Eng. Sci. 2004, 44, 1220-1230.

41. Schwarzl, F.R. Numerical calculation of stress relaxation modulus from dynamic data for linear viscoelastic materials. Rheol. Acta 1975, 14, 581-590.

(C) 2015 by the authors; licensee MDPI, Basel, Switzerland. This article is an open access article distributed under the terms and conditions of the Creative Commons Attribution license (http://creativecommons.org/licenses/by/4.0/). 